\title{
Use of the Real Time Polymerase Chain Reaction Method to Establish a Tightly Regulated Ecdysone Inducible System in Mammalian Cells
}

\author{
R. Lai ${ }^{1}$, H. Aung ${ }^{1}$, T. Walsh ${ }^{2}$ and R. Barnard ${ }^{*}$ \\ ${ }^{I}$ The School of Molecular and Microbial Sciences, The University of Queensland, Brisbane, Australia \\ ${ }^{2}$ The School of Life Science, Queensland University of Technology, Brisbane, Australia
}

\begin{abstract}
Introduction: The ecdysone inducible system potentially allows the study of conditional expression of the exogenous reporter genes that may be cell lethal or alter the phenotype during the selection of transfectants. The system relies on two independent transfections of plasmids named pVgRXR and pIND. Disruption of the regulatory element within the plasmid during stable integration can result in silenced or high background expression of the exogenous reporter gene. Previous studies [1] have reported a transient luciferase reporter assay to screen the cell lines stably transfected with pVgRXR plasmid. However, there is no suitable method to screen the subsequent pIND transfection. In this study, we demonstrate a real time polymerase chain reaction (PCR) strategy to screen for background expression problems associated with the ecdysone expression system and to simultaneously allow discrimination between the products of endogenously expressed and transfected genes.
\end{abstract}

Method: Two screening methods were applied sequentially in order to establish a functional ecdysone expression system. Firstly, the HCT116/VgRXR\#8 (a human colon cancer cell line stably transfected with pVGRXR plasmid) was established by utilising the previously reported luciferase reporter assay [1]. Finally, the functional ecdysone system (HCT116/VgRXR\#8/mutant p53) was established by a real time PCR screening strategy. This PCR based screening method for the exogenous reporter gene was made possible by utilizing the unique BGH polyA tail from the exogenous reporter gene.

Result: Even when the same parental cell line (HCT116/VgRXR\#8) was used in the subsequent transfection, background expression was still a common phenomenon. This can be monitored by efficient and sensitive real time PCR. Furthermore, the primers designed in this study have high specificity for the exogenous reporter gene.

Conclusion: The combined use of luciferase and real time PCR methods was necessary to enable the establishment of a tightly regulated ecdysone inducible system in mammalian cells.

\section{BACKGROUND}

Inducible promoter expression systems such as tetracycline and ecdysone inducible systems, have allowed the study of conditional expression of genes that may be cell lethal or alter the phenotype during the selection of transfectants $[2,3]$. The tetracycline inducible system has been reported to have a relatively high background of expression in the uninduced state. The ecdysone system was developed to address this issue [3]. In brief, the ecdysone inducible system takes advantage of the chimeric protein composed of the of the VP16 activation domain fused to an ecdysone receptor (VgECR) that specifically heterodimerizes with the retinoid $\mathrm{X}$ receptor (RXR) and binds to the unique synthetic response element $5 \mathrm{xE} / \mathrm{GRE}$ which is not recognized by endogenous nuclear hormone receptors. Under the induction of ecdysone analog, ponasterone $\mathrm{A}$, the $\mathrm{VgEcR} / \mathrm{RXR}$ dimerizes and induces the expression of the reporter genes in mammalian cells (Fig. 1). The technical difficulty associated with this system is the requirement to introduce two vectors into the experimental cell system; one carrying the VgECR and RXR

*Address correspondence to this author at the School of Molecular and Microbial Sciences, The University of Queensland, Brisbane, Australia; E-mail: rossbarnard@uq.edu.au gene (pVgRXR) and the other one carrying the response construct (pIND). Disruption of the regulatory elements within either of the plasmids during the stable integration could result in either silenced or high background levels of expression.

A previous study [1] has developed the transient luciferase reporter assay to screen the parental cell line that stably expresses a high level of VgEcR and RXR (pVgRXR). However, there is no suitable method for screening the daughter cells which have been stably transfected with exogenous gene of interest. This is critical, because in our hands we have found a high proportion of the daughter cells exhibiting background expression in uninduced states, which may be attributable to position of integration effects. A common method to screen the expression of the exogenous gene is western blotting. However, there are limitations associated with this method. One limitation is the poor affinity of antibodies for mutant forms of an expressed protein even though they may have acceptable affinity for the wild type. Conversely a western blot in some cases cannot distinguish between mutant and wild type endogenously expressed proteins. Moreover, antibodies are simply not readily available to many proteins of interest. 

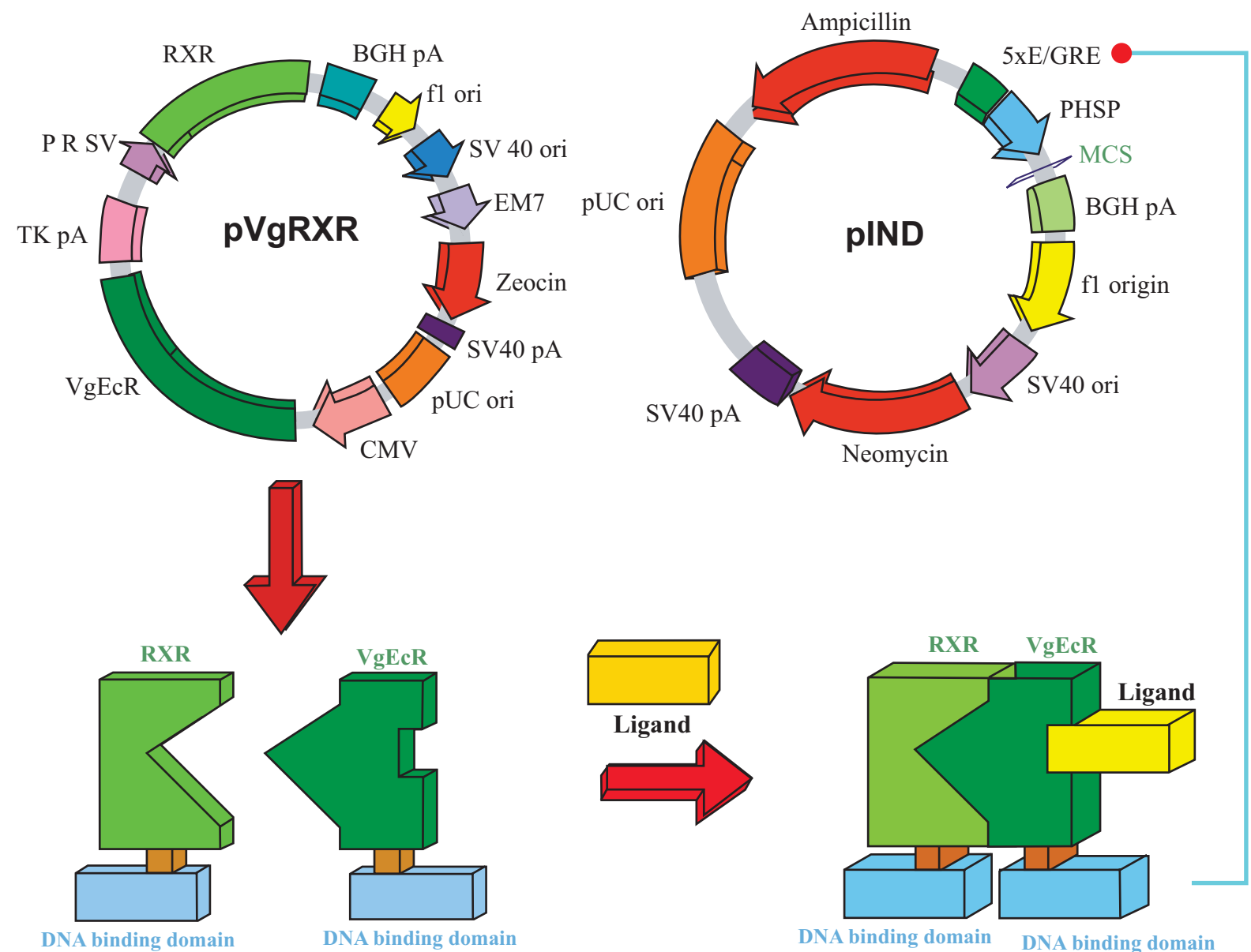

Fig. (1). The schematic representation of the ecdysone system. It contains two plasmids in the system. The pVgRXR continuously express the retinoid $x$ receptor $(\mathrm{RXR})$ and ecdysone receptor $(\mathrm{VgEcR})$. In the presence of the ligand (ponasterone $\mathrm{A}$ ) the RXR and VgEcR dimerize and reform a complex structure. This heterodimeric complex then binds to the response element, 5xE/GRE and results in transcriptional activation of the gene inserted in the multiple cloning site (MCS) within the pIND plasmid.

In this study, we demonstrate a precise real time polymerase chain reaction (PCR) strategy to screen for the background expression problem associated with the ecdysone expression system. Furthermore, this strategy is also able to overcome the limitations with the western blotting screening. The strategy utilizes real time PCR primers that recognize the unique bovine growth hormone $(\mathrm{BGH})$ polyadenylation (poly A) signal incorporated into the mRNA of the exogenous reporter gene. When combined with the previously described firefly reporter system [1], this method enabled the establishment of tightly regulated expression in a human cell line.

\section{RESULTS}

\section{Establish the Parental Cell Line (HCT116/pVGRXR)}

To establish the parental cell line, HCT116 cells were transfected with the pVgRXR plasmid. In order to identify the colonies stably expressing the functional $\mathrm{VgEcR}$ and RXR protein, we employed the luciferase reporter system [1]. After three weeks of zeocin selection, 12 colonies were selected at random and transiently transfected with the luciferase reporter gene (pIND/luciferase). After 24 hours of ponasterone A induction, the luciferase activity assay result showed widely different inducibility among different colonies (Fig. 2). In particular, clone number 8 exhibited highest inducibility (51 fold induction after 24 hours treatment with $10 \mu \mathrm{M}$ of ponasterone A), which was similar to the best observation in a previous study (55 fold induction after 24 hours treatment with $10 \mu \mathrm{M}$ of ponasterone A). This clone (HCT116/pVgRXR\#8) was then further expanded for stable transfection with pIND/R175H (p53 mutants).

\section{Background Expression is a Common Phenomenon in the Ecdysone System}

To establish the ecdysone system, the parental cell line (HCT116/pVgRXR\#8) was subsequently transfected with the $\mathrm{pIND} / \mathrm{p} 53$ plasmid. The geneticin selection allowed selection of the clones that carried the transfected p53 mutant. After 3 weeks of geneticin selection, 6 independent colonies (HCT116/VgRXR\#8/R175H) were picked up randomly for the real time PCR study. The colonies (HCT116/VgRXR\#8 $\mathrm{R} 175 \mathrm{H})$ were expanded and treated either with or without $10 \mu \mathrm{M}$ of ponasterone A for 24 hours before RNA extraction. Since the HCT116 cells expressed endogenous WT p53, in order to distinguish between the endogenous WT and transfected mutant RNA transcripts, we employed real time PCR using a reverse primer with different specificity (complementary to the unique BGH polyA sequence in the $\mathrm{pIND} / \mathrm{p} 53$ plasmid construct). The results for real time PCR amplification showed that, after 24 hours of the ponasterone A treatment, most colonies showed increased level of exoge- 


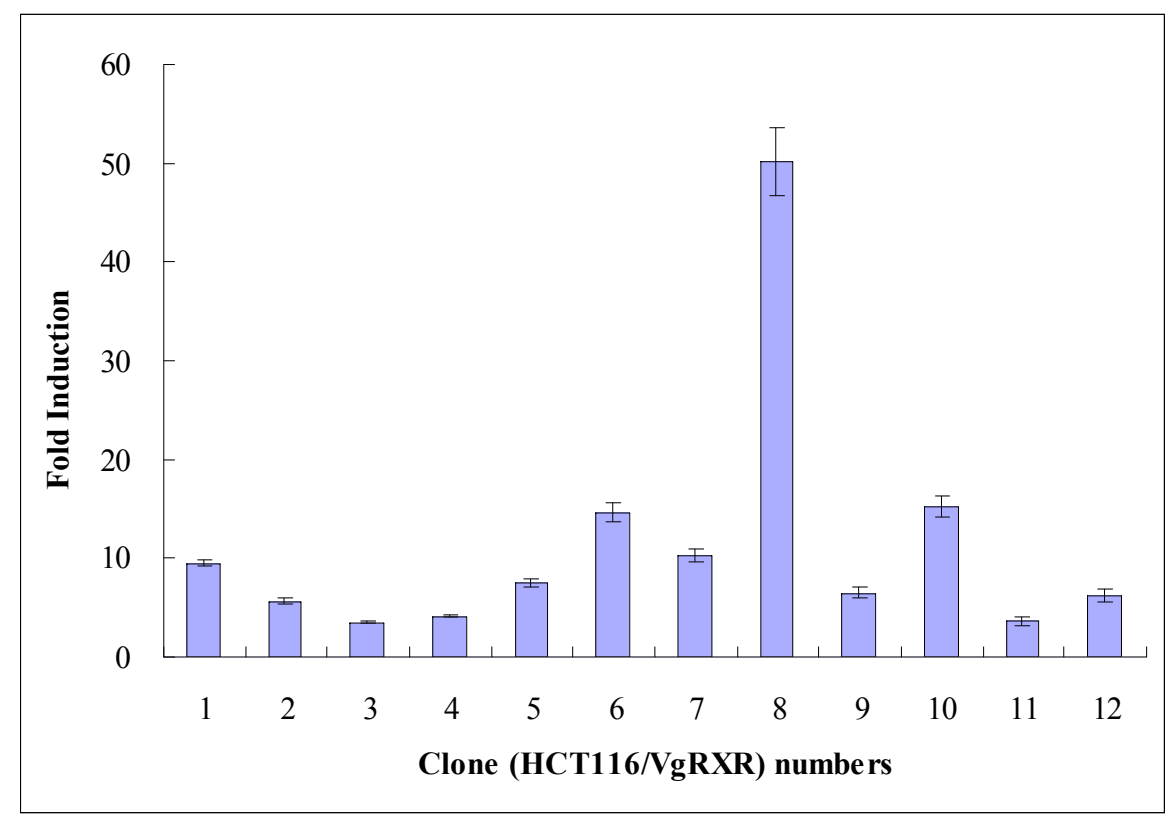

Fig. (2). The luciferase reporter gene induction in the HCT116 cells. The colonies (HCT116/vGXRX) were transiently transfected with pIND/Luciferase plasmid for 24 hours in the presence or absence of the ponasterone A. The luciferase activities of the cell lines were determined by the luminometer. All the cell lines exhibited different levels of induction. Among these stable cell lines, clone number 8 exhibited the highest luciferase induction and was selected for subsequent transfection with p53 constructs. The error bars show standard errors from the mean of three determinations.

nous mutant p53 transcript. However, these colonies (HCT116/VgRXR \#8/R175H) also exhibited widely different levels of amplification signals in the uninduced state (Fig. 3). This result clearly demonstrates that background expression is a common phenomenon associated with the ecdysone system, even when the same parental cell line is used as target for subsequent transfection.

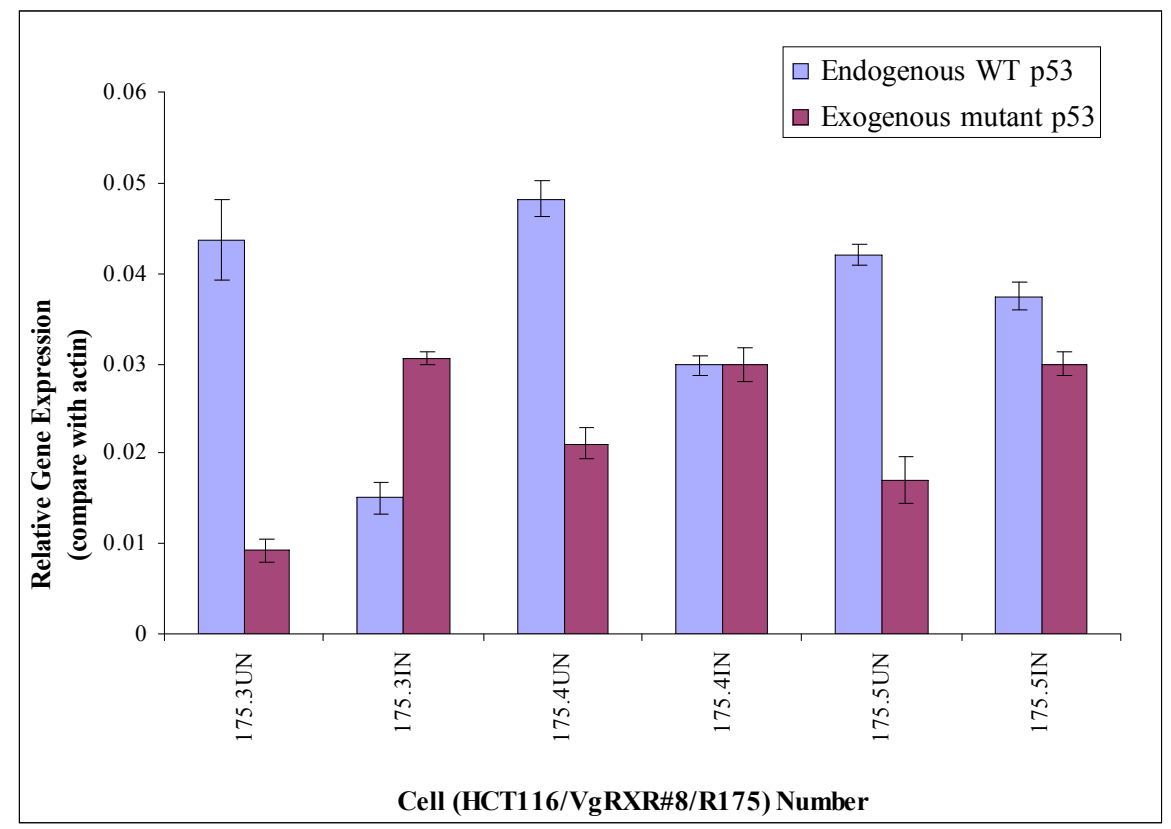

Fig. (3). The real time PCR screening result for HCT116 cell lines (HCT116/VgRXR\#8/R175H). After three weeks of geneticin selection, 6 colonies (175.1-175.6) were randomly picked up for the real time PCR study. By using different set of the reverse primers, the system was able to distinguish betweentransfected and endogenous p53. Among different daughter cell lines, each of them exhibited some inducibility after 24 hours treatment with ponasterone A (IN). Moreover, each of the cell line exhibited different levels of background expression in the absence of ponasterone A (UN). N.B. the result for cell lines 175.1 and 175.2 are not included due to zero signal detected for transfected mutant p53 after 24 hours ponasterone A induction. However, the signal for endogenous p53 is still detectable. The error bars show standard errors from the mean of three determinations. 
The BGH Poly-A Real Time PCR Primer Offers High Specificity for Exogenous Reporter Gene

In order to examine the specificity of the real time PCR primer, additional RNAs were used in the experiment, these include HCT116+/+ (WTp53), HCT116+/+ (VgRXR\#8), NCI-H358 (human lung cancer cell with a spontaneous p53 deletion), HCT 116 (p53 knockout by homologous recombination). In terms of specificity of the PCR primers, when the endogenous p53 reverse primer was used, the real time result showed strong amplification signal for endogenous WT p53 RNA in the HCT116+/+ and HCT116+/+(VgRXR\#8) but no signal was detected in the HCT116-/- and NCI-H358 cells. In the other hand, when BGH polyA reverse primer was used, the transfected p53 mutant RNA signal was only detectable in the transfectedHCT116+/+ (HCT116/VgRXR\#8/ R175H) but not in HCT116+/+, HCT116+/+ (VgRXR\#8), HCT116-/- and NCI-H358 (Fig. 4). These results indicate that the primers designed for the real time PCR have high specificity to either endogenous WT p53 or endogenous mutant $\mathrm{p} 53$ respectively.

\section{DISCUSSION}

In this study, the exogenous mutant TP53 gene was expressed in the HCT116 cell which also expressed endogenous wild type TP53 gene (GenBank accession number AF307851). The expression of mutant $\mathrm{p} 53$ protein is regulated by the ecdysone expression system.

The ecdysone inducible expression system is associated with potential background expression problems. From the same parental cell line (HCT116/VgRXR\#8), all the transfected daughter cell lines (HCT116/VgRXR\#8/R175H) ex- hibited similar RNA expression level in the induced state but different RNA expression level in uninduced states. This background expression seems to be a common phenomenon. The possible reason for the different background expression is that the transcriptional activity of the pIND/R175H construct may be subject to influences from flanking genomic sequences after stable integration [4]. From this point of view, it is very important to develop a technique which is able to monitor the transcription activity of the transfected gene after stable integration. Since the ecdysone system requires two sequential transfections of plasmids, two different reporter techniques are necessary to establish a tightly regulated ecdysone system. From the previous study, the transcription activity of the pVgRXR was monitored by using luciferase reporter system [1]. Here, we report a real time PCR method to monitor the transcriptional activity of the pIND plasmid. This screening technique is made possible by the use of the unique BGH polyadenylation signal within the pIND plasmid construct (Fig. 1). The BGH polyadenylation utilizes the GU- and U-rich sequences in the 3'-flanking region which is distinct from the conventional AAUAAA hexanucleotides [5] in human.

For the realtime experiments, the expected RT-PCR product was 200 base pairs. The expression level of both exogenous mutant and endogenous wild type p53 was quantified relative to $\beta$-actin mRNA.

\section{CONCLUSION}

In conclusion, background expression is a common problem associated with the ecdysone inducible system. We have developed a real time strategy to precisely monitor this background expression. This real-time strategy would be

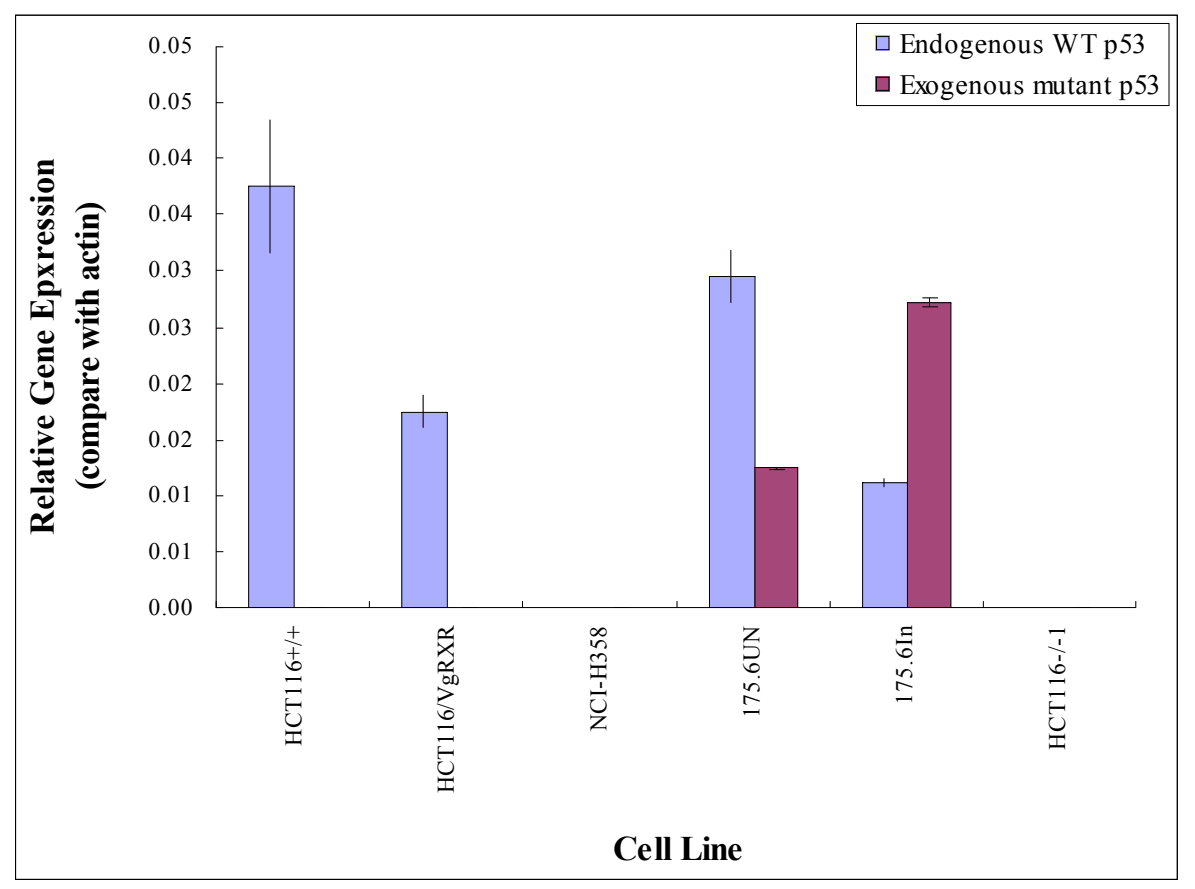

Fig. (4). The specificity of the real time PCR amplification among different cell lines. The different sets of reverse PCR primers were designed to target either endogenous p53 or transfected p53. In brief, the endogenous specific p53 reverse primer was designed targeted the 3 ' UTR region of the p53 whereas the transfected p53 specific reverse primer targeted the unique BGH polyA sequence. As a result, the endogenous signals were detected in the cell line such as HCT116+/+, HCT116/VgRXR\#8, HCT116/VgRXR\#8/175.6. The transfected p53 signal is only detected in the HCT116/VgRXR\#8/175.6 cell lines. 
particularly advantageous if the protein of interest doesn't have an antibody available or is endogenously expressed. Furthermore the real time PCR is more effective then western blotting in terms of quantifying the expression level. To our knowledge, this is also the first work to formally demonstrate and quantify the background expression problem associated with the ecdysone inducible system. Furthermore, combined with the previous study [1], these two methods (both luciferase and real time PCR methods) enable establishment of a tightly regulated ecdysone inducible system in mammalian cells.

\section{METHODS}

\section{Cell Culture}

The HCT116 human colon cancer cell lines and the NCIH358; human lung cancer cell lines were obtained from the American Type Culture Collection (Manassas, VA, USA). The HCT116 (p53-/-); human colon cancer cell line was a kind gift from Professor Bert Vogelstein (Johns Hopkins Oncology Center, the Johns Hopkins University School of Medicine, Baltimore, MD, USA) [6]. The cells were maintained in phenol red free RMPI 1640 medium (Life Technologies) supplemented with $10 \%$ heat inactivated fetal bovine serum (Life Technologies, Rockville, MD, USA) in a $5 \%$ carbon dioxide incubator at $37^{\circ} \mathrm{C}$.

\section{Establishment of Ecdysone Inducible System}

The pVgRXR plasmid (Invitrogen, Carlsbad, CA, USA) was transfected into the cells using lipofectamine plus reagent (Invitrogen Carlsbad, CA, USA). The transfection procedure was carried out according to the standard manufacturer's protocol. After 24 hours of transfection, the cells were selected in medium supplemented with $1 \mathrm{mg} / \mathrm{mL}$ of zeocin (Invitrogen, Carlsbad, CA, USA) for 3 weeks. A total of 50 individual colonies were trypsinized, seeded and expanded. Expanded samples of 12 colonies were randomly picked and transfected transiently with pIND/luciferase plasmid (Dr Osamu Tetsu, UC San Francisco, CA, USA) using lipofectamine plus reagent (Invitrogen, Carlsbad, CA, USA). All transfections were performed in triplicate. After 24 hours of transfection, cells were cultured with the medium supplemented with, or without $10 \mu \mathrm{M}$ of ponasterone $\mathrm{A}$ (Invitrogen, Carlsbad, CA, USA) for additional 24 hours. Cells were then lysed for the luciferase assay using luciferase assay system (Promega, Madison, WI, USA). The luciferase activity was determined using the Wallac TriLux (Perkin Elmer, Wellesley, MA, USA). The clone with highest inducibility was further expanded and transfected with pIND/R175H (p53 mutants). After 24 hours of transfection, the cells were selected with medium supplemented with 1.5 $\mathrm{mg} / \mathrm{mL}$ of geneticin for 3 weeks. A total of 6 clones was randomly picked and expanded for RNA extraction using Trizol reagent (Invitrogen, Carlsbad, CA, USA).

\section{RNA Sample Preparation}

Prior to RNA extraction, the HCT116/VgRXR/p53 cells were cultured for 24 hours in the presence or absence of 10 $\mu \mathrm{M}$ of Ponasterone A. Total RNA was isolated from these stable transfectants using Trizol ${ }^{\circledR}$ reagent (Invitrogen, Carlsbad, CA, USA) according to the manufacturer's protocol. Cells at approximately $90 \%$ confluency were harvested for the procedure. The concentration and purity of RNA templates were determined by measuring the $\mathrm{A}_{260}$ (absorbance measured at $260 \mathrm{~nm}$ on Smart Spec 2000, BIORAD, Hercules, CA, USA). RNA templates were treated with Dnase (Protégé, Madison, WI, USA) to avoid the possibility of false positive results. Total RNA $(1 \mu \mathrm{g})$ was reverse transcribed in a $20 \mu \mathrm{L}$ reaction with oligo dT primers (GenSet Pacific Pty Ltd, Lismore, NSW, Australia) using a superscript first strand synthesis kit (Invitrogen) according to the manufacturer's recommended protocol. For real time RT-PCR amplification, $2 \mu l$ of reverse transcribed cDNA was placed in each reaction.

\section{Real Time PCR}

For real time PCR, the specific amplification of mutant p53 gene was made possible by designing a reverse primer targeting the BGH polyadenylation site. To be able to amplify endogenous p53 specifically, reverse primer was designed based on the 3'-UTR region, because the pIND vector carries only the coding region of $\mathrm{p} 53$. The same forward primer was used to amplify both endogenous and mutant p53 sequences. The list of realtime RT-PCR primer is described Table 1.

Table 1. Real Time RT-PCR Primers for p53 Expression

\begin{tabular}{|c|c|c|c|}
\hline Name & Sequence & Size & Tm \\
\hline \hline RLp53BGHR & 5'-AGGCACAGTCGAGGCTGATC-3' & $20 \mathrm{bp}$ & $64^{\circ} \mathrm{C}$ \\
\hline RLp53UTRR & 5'-AGGCTGTCAGT GGGGAACAA-3' $^{\prime} 20 \mathrm{bp}$ & $62^{\circ} \mathrm{C}$ \\
\hline RLp53RTF & $5^{\prime}$-CCTCCCGCCATAAAAAACTCA-3' & $21 \mathrm{bp}$ & $56^{\circ} \mathrm{C}$ \\
\hline RLBactinF & $5^{\prime}$-CGTTACACCCTTTCTTGACAAAACC-3' & $25 \mathrm{bp}$ & $57^{\circ} \mathrm{C}$ \\
\hline RLBactinR & $5^{\prime}$-GCTGTCACCTTCACCGTTCCA-5' & $21 \mathrm{bp}$ & $57^{\circ} \mathrm{C}$ \\
\hline
\end{tabular}

Five different primers were used in the RT-PCR study. The RLp53BGHR primer was designed to anneal to the BGH poly A sequence of the exogenous p53 gene. The RLp53UTRR primer was designed to anneal to the 3'-UTR region of the endogenous p53 gene. RLp53RTF was designed to anneal to the 3' end of the coding region of both the endogenous and the exogenous p53 gene. The RLBactinF and RLBactinR were designed to amplify the house-keeping gene, $\beta$-actin.

The mRNA expression of the exogenous and the endogenous p53 was quantified by real time RT-PCR on the ABI PRISM 7700 Sequence Detection System (Applied Biosystems, Foster City, CA, USA). PCR was performed using SYBR Green PCR reagent (Applied Biosystems) according to the manufacturer's instructions. PCR cycles consisted of an initial denaturing step at $95^{\circ} \mathrm{C}$ for $10 \mathrm{~min}$, followed by 45 cycles at $95^{\circ} \mathrm{C}$ for $15 \mathrm{sec}$ and $60^{\circ} \mathrm{C}$ for $1 \mathrm{~min}$. A standard curve was constructed with a plasmid containing the same fragment amplified by realtime PCR. The relative expression in each sample was calculated with respect to the standard calibration curve. Normalization of cDNA load was performed against the housekeeping gene $\beta$-actin. Each sample was analyzed twice, and each PCR experiment included two nontemplated control wells. PCR products were confirmed as single bands using electrophoresis.

The relative amount of the p53 RNA expression was defined by the $\Delta \mathrm{Ct}$ method as described by Livak [7]. The formula was applied as follows:

$$
\text { Relative expression units }=2^{-\Delta \mathrm{Ct}}
$$

where $\Delta \mathrm{Ct}=(\mathrm{Ct}$ endogenous or exogenous $\mathrm{p} 53)-(\mathrm{Ct} \beta-$ actin). 


\section{ABBREVIATIONS}

$$
\begin{array}{ll}
\mathrm{PCR} & =\text { Polymerase Chain Reaction } \\
\mathrm{BGH} & =\text { Bovine Growth Hormone } \\
\text { Poly A } & =\text { Polyadenylation }
\end{array}
$$

\section{ACKNOWLEDGEMENTS}

We are indebted to Dr Osamu Tetsu (University of California, San Francisco, CA, USA) for the gift of the pIND/luciferase construct and Professor Bert Vogelstein (Johns Hopkins Oncology Center, The Johns Hopkins University, School of Medicine, Baltimore, MD, USA) for the kind gift of the HCT116 cell line.

\section{Authors' Contributions}

R. Lai has prepared the manuscript and performed all the primer design and experimental work except the real-time
PCR. H. Aung performed the real time PCR. T. Walsh and $R$. Barnard gave suggestions for experimental design and helped in preparing and revising the manuscript.

\section{REFERENCES}

[1] Wakita, K.; McCormick, F.; Tetsu, O. Biotechniques, 2001, 31, 414.

[2] No, D.; Yao, T.P.; Evans, R.M. Proc. Natl. Acad. Sci. USA, 1993, 93, 3346 .

[3] Saez, E.; No, D.; West, A.; Evans, R.M. Curr. Opin. Biotechnol., 1997, 5, 608 .

[4] Gram, G.J.; Nielsen, S.D.; Hansen, J.E. J. Hematother., 1998, 7, 333.

[5] Goodwin, E.C.; Rottman, F.M. J. Biol. Chem., 1992, 267, 16330.

[6] Bunz, F.; Dutriaux, A.; Lengauer, C.; Waldman, T.; Zhou, S.; Brown, J.P.; Sedivy, J.M.; Kinzler, K.W.; Vogelstein, B. Science, 1998, 282, 1497.

[7] Livak, K.J. PE-ABI Sequence Detector User Bull., 1997, 2.

Received: April 21, 2008

Revised: May 7, 2008

Accepted: May 14, 2008

(C) Lai et al.; Licensee Bentham Open.

This is an open access article distributed under the terms of the Creative Commons Attribution License (http://creativecommons.org/licenses/by/2.5/), which permits unrestrictive use, distribution, and reproduction in any medium, provided the original work is properly cited. 